\title{
A formula for the number of spanning trees of a multi-star related graph
}

\author{
Wen-Ming Yan ${ }^{\mathrm{a}, 1}$, Wendy Myrvold ${ }^{\mathrm{b}, 2}$, Kuo-Liang Chung ${ }^{\mathrm{c}, *}$ \\ a Department of Computer Science and Information Engineering, National Taiwan University, Taipei, Taiwan 10764 \\ b Department of Computer Science, University of Victoria, P.O. Box 3055, STN CSC, Victoria, BC, Canada V8W $3 P 6$ \\ ${ }^{c}$ Department of Information Management and Institute of Information Engineering, National Taiwan University of Science and Technology, \\ No. 43, Section 4, Keelung Road, Taipei, Taiwan 10672
}

Received 1 July 1998; received in revised form 1 October 1998

Communicated by S.G. Akl

\begin{abstract}
Using a new labeling technique and matrix computations, this paper derives a closed formula for the number of spanning trees of a multi-star related graph $G=K_{n}-K_{m}\left(a_{1}, a_{2}, \ldots, a_{m}\right)$, where $K_{m}\left(a_{1}, a_{2}, \ldots, a_{m}\right)$ consists of $m$ star graphs such that the $i$ th one has a root node connected to $a_{i}$ leaves, and further, the $m$ roots are connected together to form a complete graph. This result generalizes the previous result by Nikolopoulos and Rondogiannis (1998) which is limited to $m=2,3,4$. 1998 Elsevier Science B.V. All rights reserved.
\end{abstract}

Keywords: Combinatorial problems; Spanning trees; Multi-star graph

\section{Introduction}

An undirected graph $G$ consist of a set $V(G)$ of vertices and a set $E(G)$ of edges where each edge corresponds to an unordered pair of vertices from $V(G)$. All graphs considered in this paper are simple (they have no loops or multiple edges), finite, and undirected.

A complete graph on $n$ vertices, denoted $K_{n}$, has one edge between each pair of distinct vertices. The complement $\bar{G}$ of a simple graph $G=(V, E)$ on $n$

\footnotetext{
* Corresponding author. Email: klchung@cs.ntust.edu.tw. Supported by NSC88-2213-E011-005/006.

${ }^{1}$ Email: ganboon@csie.ntu.edu.tw. Supported by NSC87-2119M002-006.

${ }^{2}$ Email: wendym@csr.uvic.ca. Supported by NSERC.
}

vertices is the $n$-vertex graph containing exactly the edges of $K_{n}$ which are not in $G$.

A multi-star related graph, $G=K_{n}-K_{m}\left(a_{1}, a_{2}\right.$, $\left.\ldots, a_{m}\right)$, is an $n$-vertex graph whose complement consists of $K_{m}$ with vertices labeled $v_{1}, v_{2}, \ldots, v_{m}$, plus there are $a_{i}$ vertices of degree one (leaves) incident to vertex $v_{i}$ of the $K_{m}$, and the remaining $k=n-m-a_{1}-a_{2}-\cdots-a_{m}$ vertices are isolated points. Fig. 1 illustrates a triple-star graph $K_{3}(3,2,3)$.

Recently, using matrix computations, Nikolopoulos and Rondogiannis [3] derived a closed form for the number of spanning trees of multi-star related graphs $G=K_{n}-K_{m}\left(a_{1}, a_{2}, \ldots, a_{m}\right)$ for $m=2,3,4$. Surprisingly, some previous closed forms $[1,2,4,5]$ for counting the number of spanning trees of some special graphs are covered in their result. 


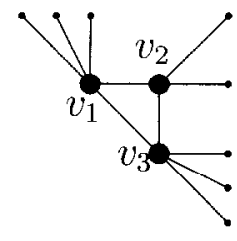

Fig. $1 . K_{3}(3,2,3)$.

Using a new labeling technique and matrix computations, this paper derives a closed formula for the number of spanning trees of $G=K_{n}-K_{m}\left(a_{1}, a_{2}\right.$, $\left.\ldots, a_{m}\right)$ for arbitrary $m$. This extends the previous result [3] which is limited to $m=2,3,4$.

\section{Counting spanning trees}

The complement spanning tree matrix [5] $C$ for a graph $G$ is defined by

$C_{i j}= \begin{cases}n-\bar{d}_{i} & \text { if } i=j, \\ e_{i j} & \text { if } i \neq j,\end{cases}$

where $\bar{d}_{i}$ is the degree of vertex $i$ in $\bar{G}$ and $e_{i j}$ is one if $(i, j)$ is in $E(\bar{G})$ and 0 otherwise.

The following result first derived by Temperley [5] provides a formula for the number of spanning trees of a graph. The notation $|C|$ represents the determinant of matrix $C$.

Theorem 1 [5]. For the graph $G$, the number of spanning trees of $G, \tau(G)$, is equal to $\left(1 / n^{2}\right)|C|$.

This is applied in the next sections to derive a closed formula for the number of spanning trees of a multistar related graph.

\section{The generalization}

In this section, a new approach to labeling the graph $G$ is presented first. Then the closed form for counting the number of spanning trees of $G$ for arbitrary $m$ is given.

\subsection{The new labeling method}

Given the graph $G=K_{n}-K_{m}\left(a_{1}, a_{2}, \ldots, a_{m}\right)$, the labeling method used by Nikolopolous and Rondogiannis [3] is to label the nodes in $V\left(K_{n}\right)-$

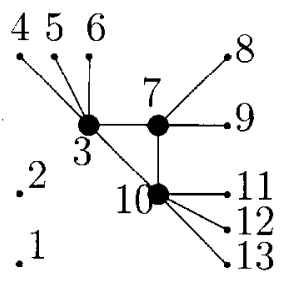

Fig. 2. Labeling the complement graph of $K_{13}-K_{3}(3,2,3)$ [3].

$V\left(K_{m}\left(a_{1}, a_{2}, \ldots, a_{m}\right)\right)$ first, then they label the nodes in the first star graph with $a_{1}+1$ nodes. Finally, they label the nodes in the $m$ th star graph with $a_{m}+1$ nodes. Therefore, using their labeling method, the labeling of the complement of $K_{13}-K_{3}(3,2,3)$ is shown in Fig. 2. In addition, the complement spanning tree matrix of $K_{13}-K_{3}(3,2,3)$ is given by

$C=\left(\begin{array}{ll}n I_{2} & \\ & D\end{array}\right)$,

where $n=13, m=3$, and

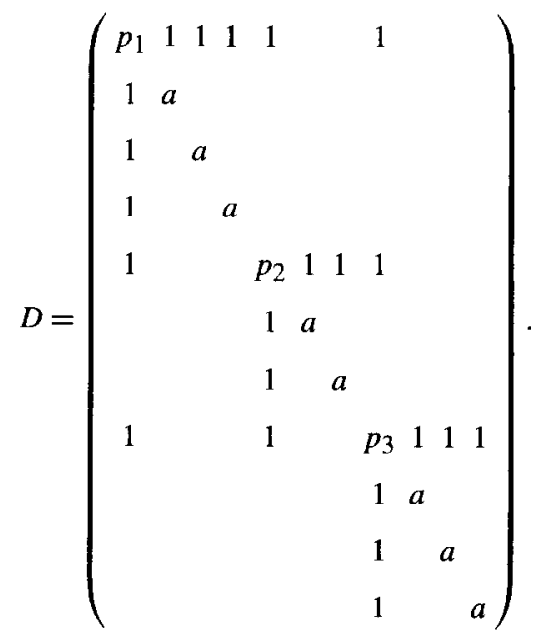

In matrix $D, a=n-1=12$ and $p_{i}=n-a_{i}-m+$ $1=11-a_{i}$ for $1 \leqslant i \leqslant 3$.

Our labeling method for $G=K_{n}-K_{m}\left(a_{1}, a_{2}, \ldots\right.$, $\left.a_{m}\right)$ is to label the nodes in $V\left(K_{n}\right)-V\left(K_{m}\left(a_{1}, a_{2}\right.\right.$, $\left.\ldots, a_{m}\right)$ ) first, secondly to label the roots of the $m$ star graphs. Then we label the nodes in the first star graph except the root node, and so on. Using the same graph as shown in Fig. 2, the new labeling is shown in Fig. 3. 


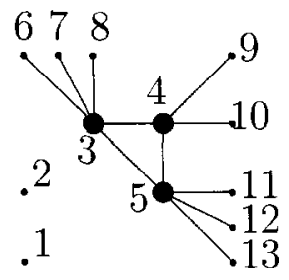

Fig. 3. The new labeling of the vertices of $K_{13}-K_{3}(3,2,3)$.

The complement spanning tree matrix corresponding to the graph as labeled in Fig. 3 is given by

$$
C=\left(\begin{array}{ll}
n I_{2} & \\
& D
\end{array}\right) \text {, }
$$

but now

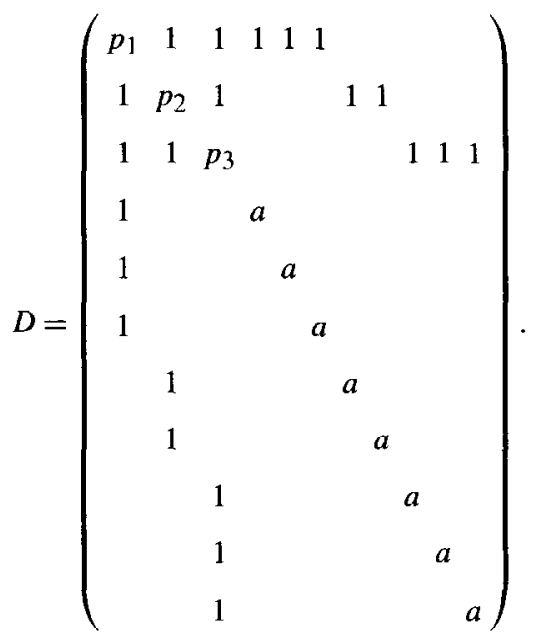

The values of $p_{i}$ and $a$ are as defined before.

Our new labeling method transforms the matrix $D$ into a kite form. This simplifies the algebra involved in determining a closed formula for the number of spanning trees of $G=K_{n}-K_{m}\left(a_{1}, a_{2}, \ldots, a_{m}\right)$ for arbitrary $m$.

\subsection{The closed formula}

The next theorem gives our new formula. The proof involves algebraic manipulations of the complement spanning tree matrix which are facilitated by use of the new labeling scheme described in the previous section.

Theorem 2. The number of spanning trees of a multistar related graph $G=K_{n}-K_{m}\left(a_{1}, a_{2}, \ldots, a_{m}\right)$,
$\tau(G)=n^{k-2} *(n-1)^{a_{1}+a_{2}+\cdots+a_{m}}\left[1+1 /\left(q_{1}-1\right)+\right.$ $\left.1 /\left(q_{2}-1\right)+\cdots+1 /\left(q_{m}-1\right)\right]\left(q_{1}-1\right)\left(q_{2}-1\right) \cdots\left(q_{m}-\right.$ 1) where $q_{i}=n-a_{i}-m+1-a_{i} /(n-1)$, and $k=n-m-a_{1}-a_{2}-\cdots-a_{m}$.

Proof. If the graph is labeled by the new method as described in the previous section, then the complement spanning tree matrix of $G$ is given by

$C=\left(\begin{array}{ll}n I_{k} & \\ & D\end{array}\right)$,

where $k=n-m-a_{1}-a_{2}-\cdots-a_{m}$ and the matrix $D$ is of a kite form and is written as

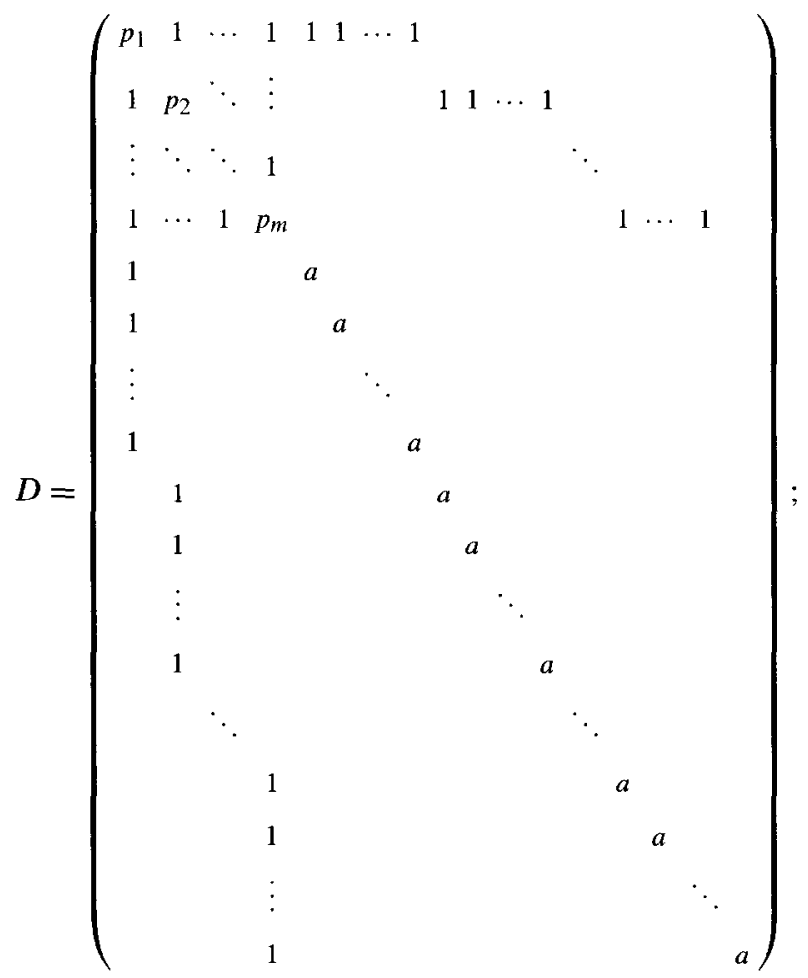

where $a=n-1$ and $p_{i}=n-a_{i}-m+1$ for $1 \leqslant$ $i \leqslant m$. By Theorem 1 , the number of spanning trees is $n^{k-2}|D|$.

To simplify the computation of the determinant of $D$, we first zero out the ones in columns 1 to $m$ which occur in the rows indexed by $m+1$ or higher as follows: the one in row $i$ is zeroed out by adding $-1 /(n-1)$ times column $i$. This affects only the main diagonals in the first $m$ columns of the matrix. 
The matrix now has an $m$ by $m$ block which we label $Q$ in the upper left hand corner with ones on the off-diagonal and diagonal entries $q_{i}=n-a_{i}$ $m+1-a_{i} /(n-1)$. In columns 1 to $m$, the entries in the rows indexed $m+1$ or more are all zeroes. The determinant of $D$ equals $(n-1)^{a_{1}+a_{2}+\cdots+a_{m}}|Q|$.

The matrix $Q$ has diagonal elements $q_{1}, q_{2}, \ldots, q_{m}$ and has ones on the off-diagonal. Border this matrix by adding a new first row and column. All the entries in the new row are ones. The entries in the new column which are not in the new row are zeroes. From expanding about the new column, it is clear that this new matrix has the same determinant as $Q$.

Subtract the new row from each of the other rows. The diagonal entries in the old row indexed $i$ are now $q_{i}-1$. The first column has all -1 's except for the one in the first row. The first row is all ones. The remaining elements of the matrix are zero. Now add to the first column $1 /\left(q_{i}-1\right)$ times each of the other columns to zero out the off-diagonal entries in this column. The entry in the first row and column becomes $1+1 /\left(q_{1}-1\right)+1 /\left(q_{2}-1\right)+\cdots+1 /\left(q_{m}-1\right)$. Also, the matrix is now upper triangular, so the determinant is: $\left[1+1 /\left(q_{1}-1\right)+1 /\left(q_{2}-1\right)+\cdots+1 /\left(q_{m}-\right.\right.$ 1)] $\left(q_{1}-1\right)\left(q_{2}-1\right) \cdots\left(q_{m}-1\right)$.

In summary, $\tau(G)$ is equal to $n^{k-2}|D|$. But $|D|$ is equal to $(n-1)^{a_{1}+a_{2}+\cdots+a_{m}}|Q|$. Further, $|Q|=[1+$ $\left.1 /\left(q_{1}-1\right)+1 /\left(q_{2}-1\right)+\cdots+1 /\left(q_{m}-1\right)\right]\left(q_{1}-\right.$ 1) $\left(q_{2}-1\right) \cdots\left(q_{m}-1\right)$. Thus, $\tau(G)=n^{k-2}(n-$ $1)^{a_{1}+a_{2}+\cdots+a_{m}}\left[1+1 /\left(q_{1}-1\right)+1 /\left(q_{2}-1\right)+\cdots+\right.$ $\left.1 /\left(q_{m}-1\right)\right]\left(q_{1}-1\right)\left(q_{2}-1\right) \cdots\left(q_{m}-1\right)$ as required.

\section{Conclusion}

We have described how to derive the closed form for the number of spanning trees of a multi-star related graph $G=K_{n}-K_{m}\left(a_{1}, a_{2}, \ldots, a_{m}\right)$ for arbitrary $m$. Our new labeling method transforms the complement spanning tree matrix $G$ into matrix whose left-upper submatrix is an identity matrix and whose right-lower submatrix is of a kite form that makes the derivation of the closed form easier. The main contribution of this paper is that based on our new labeling method, the proposed closed form for arbitrary $m$ generalizes the result of Nikolopoulos and Rondogiannis [3] whose closed form is limited to $m=2,3$, and 4 .

\section{References}

[1] C. Berge, Graphs and Hypergraphs, North-Holland, Amsterdam, 1973.

[2] W. Moon, Enumerating labeled trees, in: F. Harary (Ed.), Graph Theory and Theoretical Physics, Academic Press, London, 1967, pp. 261-271.

[3] S.D. Nikolopoulos, P. Rondogiannis, On the number of spanning trees of multi-star related graphs, Inform. Process. Lett. 65 (1998) 183-188.

[4] P.V. O'Neil, The number of trees in a certain network, Notices Amer. Math. Soc. 10 (1963) 569.

[5] H.N.V. Temperley, On the mutual cancellation of cluster integrals in Mayer's fugacity series, Proc. Phys. Soc. 83 (1964) 3 16. 